\title{
Hamiltonian structure of the guiding-center Vlasov-Maxwell equations
}

\author{
Alain J. Brizard \\ Department of Physics, Saint Michael's College, Colchester, VT 05439, USA
}

(Dated: September 9, 2021)

\begin{abstract}
The Hamiltonian structure of the guiding-center Vlasov-Maxwell equations is presented in terms of a Hamiltonian functional and a guiding-center Vlasov-Maxwell bracket. The bracket, which is shown to satisfy the Jacobi identity exactly, is used to show that the guiding-center momentum and angular-momentum conservation laws can also be expressed in Hamiltonian form.
\end{abstract}

\section{INTRODUCTION}

The complementary Lagrangian and Hamiltonian formulations of dissipationless plasma equations have a rich history in plasma physics 1, 2]. In the Lagrangian formulation for the Vlasov-Maxwell equations, for example, one of several types of variational (Euler, Lagrange, or Euler-Poincaré) principles is used to derive these dissipationless plasma equations [3, 4], while their exact conservation laws can be derived through the Noether method.

In the Hamiltonian formulation of dissipationless plasma equations, on the other hand, the equations for a given set of plasma fields $\boldsymbol{\psi}$ are, first, written in Hamiltonian form:

$$
\frac{\partial \psi^{a}}{\partial t}=\mathrm{J}^{a b} \circ \frac{\delta \mathcal{H}}{\delta \psi^{b}}
$$

in terms of a Hamiltonian functional $\mathcal{H}[\boldsymbol{\psi}]$ and the antisymmetric Poisson operator $\mathrm{J}^{a b}(\boldsymbol{\psi})$ acting (denoted as ०) on the functional derivative $\delta \mathcal{H} / \delta \psi^{b}$. Next, the evolution of an arbitrary functional $\mathcal{F}[\boldsymbol{\psi}]$ is expressed in Hamiltonian form

$$
\frac{\partial \mathcal{F}}{\partial t}=\left\langle\frac{\delta \mathcal{F}}{\delta \psi^{a}} \mid \frac{\partial \psi^{a}}{\partial t}\right\rangle=\left\langle\frac{\delta \mathcal{F}}{\delta \psi^{a}} \mid \mathrm{J}^{a b} \circ \frac{\delta \mathcal{H}}{\delta \psi^{b}}\right\rangle \equiv[\mathcal{F}, \mathcal{H}],
$$

which is then used to construct a bracket [, ] on functionals, where the inner product $\langle\mid\rangle$ involves suitable integrations over the domains of the field components.

For example, we consider the Vlasov-Maxwell equations with field components $\boldsymbol{\psi}=(f, \mathbf{E}, \mathbf{B})$, which are expressed in Hamiltonian form (11):

$$
\begin{aligned}
\frac{\partial f(\mathbf{x}, \mathbf{p}, t)}{\partial t} & =-\left\{f, \frac{\delta \mathcal{H}}{\delta f}\right\}+4 \pi q\{f, \mathbf{x}\} \cdot \frac{\delta \mathcal{H}}{\delta \mathbf{E}} \\
& \equiv \mathrm{J}^{f f} \circ \frac{\delta \mathcal{H}}{\delta f}+\mathrm{J}^{f \mathbf{E}} \circ \frac{\delta \mathcal{H}}{\delta \mathbf{E}} \\
\frac{\partial \mathbf{E}(\mathbf{x}, t)}{\partial t} & =4 \pi c \nabla \times \frac{\delta \mathcal{H}}{\delta \mathbf{B}}+4 \pi q \int_{\mathbf{p}}\{\mathbf{x}, f\} \frac{\delta \mathcal{H}}{\delta f} \\
& \equiv \mathrm{J}^{\mathbf{E B}} \circ \frac{\delta \mathcal{H}}{\delta \mathbf{B}}+\mathrm{J}^{\mathbf{E} f} \circ \frac{\delta \mathcal{H}}{\delta f}, \\
\frac{\partial \mathbf{B}(\mathbf{x}, t)}{\partial t} & =-4 \pi c \nabla \times \frac{\delta \mathcal{H}}{\delta \mathbf{E}} \equiv \mathrm{J}^{\mathbf{B E}} \circ \frac{\delta \mathcal{H}}{\delta \mathbf{E}},
\end{aligned}
$$

where the Vlasov function $f$ is defined in six-dimensional particle phase space $\mathbf{z}=(\mathbf{x}, \mathbf{p})$, while the electric and magnetic fields (E, B) are defined in three-dimensional configuration space $\mathbf{x}$. with the single-particle noncanonical Poisson bracket

$$
\{f, g\}=\nabla f \cdot \frac{\partial g}{\partial \mathbf{p}}-\frac{\partial f}{\partial \mathbf{p}} \cdot \nabla g+\frac{q}{c} \mathbf{B} \cdot \frac{\partial f}{\partial \mathbf{p}} \times \frac{\partial g}{\partial \mathbf{p}},
$$

and the Hamiltonian functional

$$
\mathcal{H}=\int_{\mathbf{z}} f \frac{|\mathbf{p}|^{2}}{2 m}+\int_{\mathbf{x}} \frac{1}{8 \pi}\left(|\mathbf{E}|^{2}+|\mathbf{B}|^{2}\right),
$$

with $\delta \mathcal{H} / \delta f=|\mathbf{p}|^{2} / 2 m, \delta \mathcal{H} / \delta \mathbf{E}=\mathbf{E} / 4 \pi$, and $\delta \mathcal{H} / \delta \mathbf{B}=$ $\mathbf{B} / 4 \pi$. In Eqs. (4) and (7), summation over particle species is implied whenever an integration over the Vlasov distribution $f$ appears, where $\int_{\mathbf{p}}$ and $\int_{\mathbf{z}}$ denote integrations over particle momentum and particle phase space, respectively, while $\int_{\mathbf{x}}$ denotes an integral over configuration space.

By expressing the evolution of an arbitrary functional $\mathcal{F}[f, \mathbf{E}, \mathbf{B}]$ on the Vlasov-Maxwell fields

$$
\begin{aligned}
\frac{\partial \mathcal{F}}{\partial t} & =\int_{\mathbf{z}} \frac{\partial f}{\partial t} \frac{\delta \mathcal{F}}{\delta f}+\int_{\mathbf{x}}\left(\frac{\partial \mathbf{E}}{\partial t} \cdot \frac{\delta \mathcal{F}}{\delta \mathbf{E}}+\frac{\partial \mathbf{B}}{\partial t} \cdot \frac{\delta \mathcal{F}}{\delta \mathbf{B}}\right) \\
& \equiv\left\langle\frac{\delta \mathcal{F}}{\delta \psi^{a}} \mid \frac{\partial \psi^{a}}{\partial t}\right\rangle=\left\langle\frac{\delta \mathcal{F}}{\delta \psi^{a}} \mid \mathrm{J}^{a b} \circ \frac{\delta \mathcal{H}}{\delta \psi^{b}}\right\rangle,
\end{aligned}
$$

we easily arrive at the Vlasov-Maxwell bracket [5-7]

$$
\begin{aligned}
\frac{\partial \mathcal{F}}{\partial t}= & \int_{\mathbf{z}} f\left\{\frac{\delta \mathcal{F}}{\delta f}, \frac{\delta \mathcal{H}}{\delta f}\right\} \\
& +4 \pi q \int_{\mathbf{z}} f\left(\frac{\delta \mathcal{H}}{\delta \mathbf{E}} \cdot\left\{\mathbf{x}, \frac{\delta \mathcal{F}}{\delta f}\right\}-\frac{\delta \mathcal{F}}{\delta \mathbf{E}} \cdot\left\{\mathbf{x}, \frac{\delta \mathcal{H}}{\delta f}\right\}\right) \\
& +4 \pi c \int_{\mathbf{x}}\left(\frac{\delta \mathcal{F}}{\delta \mathbf{E}} \cdot \nabla \times \frac{\delta \mathcal{H}}{\delta \mathbf{B}}-\frac{\delta \mathcal{H}}{\delta \mathbf{E}} \cdot \nabla \times \frac{\delta \mathcal{F}}{\delta \mathbf{B}}\right) \\
\equiv & {[\mathcal{F}, \mathcal{H}], }
\end{aligned}
$$

where integrations by parts were performed. The proof that the Vlasov-Maxwell bracket (9) satisfies the Jacobi identity for arbitrary functionals $(\mathcal{F}, \mathcal{G}, \mathcal{K})$ :

$$
[[\mathcal{F}, \mathcal{G}], \mathcal{K}]+[[\mathcal{G}, \mathcal{K}], \mathcal{F}]+[[\mathcal{K}, \mathcal{F}], \mathcal{G}]=0
$$

was given in the Appendix of Ref. [8], where the Poisson bracket (6) was broken into canonical and non-canonical parts, and Appendix B of Ref. [9], where properties of 
the full Poisson bracket (6) were used. We note that the Jacobi property of the Vlasov-Maxwell bracket (9) is inherited from the Jacobi property of the Poisson bracket (6), which requires that $\nabla \cdot \mathbf{B}=0$.

The purpose of the present paper is to follow a similar construction for the guiding-center Hamiltonian structure directly from the guiding-center Vlasov-Maxwell equations. This approach is in contrast to the Lietransform construction of a Hamiltonian structure for the reduced Vlasov-Maxwell equations [10], which is derived from the Vlasov-Maxwell bracket (9) and automatically guarantees that the reduced Vlasov-Maxwell bracket satisfies the Jacobi property. Here, while there is no guarantee that the guiding-center Vlasov-Maxwell bracket will satisfy the Jacobi property, its derivation is simple.

\section{GUIDING-CENTER VLASOV-MAXWELL EQUATIONS}

The variational formulations of the guiding-center Vlasov-Maxwell equations were presented by Pfirsch and Morrison [11] and more recently by Brizard and Tronci [12], whose works also included a derivation of exact conservation laws for energy-momentum and angular momentum through the Noether method. The guiding-center equations of motion considered here are the simplest equations derived from a variational principle [13, 14].

First, the guiding-center single-particle Lagrangian for a charged particle (of charge $q$ and mass $m$ ) moving in a reduced phase space, with guiding-center position $\mathbf{X}$ and guiding-center parallel momentum $p_{\|}$, is expressed as

$$
L_{\mathrm{gc}}=\left(\frac{q}{c} \mathbf{A}+p_{\|} \widehat{\mathrm{b}}\right) \cdot \frac{d \mathbf{X}}{d t}-\left(q \Phi+K_{\mathrm{gc}}\right),
$$

where the electromagnetic potentials $(\Phi, \mathbf{A})$ yield the electric field $\mathbf{E}=-\nabla \Phi-c^{-1} \partial \mathbf{A} / \partial t$ and the magnetic field $\mathbf{B}=\nabla \times \mathbf{A} \equiv B \widehat{\mathrm{b}}$, and the guiding-center kinetic energy is $K_{\mathrm{gc}}=p_{\|}^{2} / 2 m+\mu B$, where $\mu$ denotes the guiding-center magnetic moment (which is a guidingcenter invariant). The guiding-center equations of motion are derived from the guiding-center Lagrangian (11) as Euler-Lagrange equations, which are expressed as

$$
\begin{aligned}
\frac{d \mathbf{X}}{d t} & =\left\{\mathbf{X}, K_{\mathrm{gc}}\right\}_{\mathrm{gc}}+q \mathbf{E}^{*} \cdot\{\mathbf{X}, \mathbf{X}\}_{\mathrm{gc}} \\
\frac{d p_{\|}}{d t} & =\left\{p_{\|}, K_{\mathrm{gc}}\right\}_{\mathrm{gc}}+q \mathbf{E}^{*} \cdot\left\{\mathbf{X}, p_{\|}\right\}_{\mathrm{gc}}
\end{aligned}
$$

where the guiding-center Poisson bracket [14]

$$
\begin{aligned}
\{f, g\}_{\mathrm{gc}} \equiv & \frac{\mathbf{B}^{*}}{B_{\|}^{*}} \cdot\left(\nabla f \frac{\partial g}{\partial p_{\|}}-\frac{\partial f}{\partial p_{\|}} \nabla g\right) \\
& -\frac{c \widehat{\mathrm{b}}}{q B_{\|}^{*}} \cdot \nabla f \times \nabla g
\end{aligned}
$$

is used without the ignorable gyromotion pair $(\mu, \theta)$, and the effective fields are

$$
\left.\begin{array}{l}
\mathbf{E}^{*} \equiv \mathbf{E}-\left(p_{\|} / q\right) \partial \widehat{\mathrm{b}} / \partial t \\
\mathbf{B}^{*} \equiv \mathbf{B}+\left(p_{\|} c / q\right) \nabla \times \widehat{\mathrm{b}} \\
B_{\|}^{*} \equiv \widehat{\mathrm{b}} \cdot \mathbf{B}^{*}=B+\left(p_{\|} c / q\right) \widehat{\mathrm{b}} \cdot \nabla \times \widehat{\mathrm{b}}
\end{array}\right\}
$$

We note that the guiding-center Poisson bracket (14) satisfies the Jacobi property for arbitrary functions $(f, g, h)$ :

$$
\left\{\{f, g\}_{\mathrm{gc}}, h\right\}_{\mathrm{gc}}+\left\{\{g, h\}_{\mathrm{gc}}, f\right\}_{\mathrm{gc}}+\left\{\{h, f\}_{\mathrm{gc}}, g\right\}_{\mathrm{gc}}=0,
$$

subject to the condition

$$
\nabla \cdot \mathbf{B}^{*}=\nabla \cdot \mathbf{B}=0
$$

which is satisfied by the definition (15). We also note that the guiding-center Jacobian $\mathcal{J}_{\text {gc }} \equiv 2 \pi m B_{\|}^{*}$ satisfies the guiding-center Liouville theorem

$$
\frac{\partial \mathcal{J}_{\mathrm{gc}}}{\partial t}+\nabla \cdot\left(\mathcal{J}_{\mathrm{gc}} \frac{d \mathbf{X}}{d t}\right)+\frac{\partial}{\partial p_{\|}}\left(\mathcal{J}_{\mathrm{gc}} \frac{d p_{\|}}{d t}\right)=0
$$

with the guiding-center equations of motion (12)-(13).

Next, we introduce the guiding-center Vlasov-Maxwell equations [12] for the guiding-center fields $\boldsymbol{\Psi}_{\mathrm{gc}}=$ $\left(F_{\mathrm{gc}}, \mathbf{E}, \mathbf{B}\right)$ :

$$
\begin{aligned}
\frac{\partial F_{\mathrm{gc}}}{\partial t} & =-\nabla \cdot\left(F_{\mathrm{gc}} \frac{d \mathbf{X}}{d t}\right)-\frac{\partial}{\partial p_{\|}}\left(F_{\mathrm{gc}} \frac{d p_{\|}}{d t}\right) \\
\frac{\partial \mathbf{E}}{\partial t} & =c \nabla \times\left(\mathbf{B}-4 \pi \mathbf{M}_{\mathrm{gc}}\right)-4 \pi q \int_{P} F_{\mathrm{gc}} \frac{d \mathbf{X}}{d t} \\
& \equiv c \nabla \times \mathbf{H}_{\mathrm{gc}}-4 \pi \mathbf{J}_{\mathrm{gc}} \\
\frac{\partial \mathbf{B}}{\partial t} & =-c \nabla \times \mathbf{E}
\end{aligned}
$$

where the phase-space density $F_{\mathrm{gc}} \equiv F \mathcal{J}_{\mathrm{gc}}$ is defined in terms of the guiding-center Vlasov function $F$ and the guiding-center Jacobian $\mathcal{J}_{\text {gc }}$, the guiding-center momentum integral $\int_{P} \equiv \int d p_{\|} d \mu$ excludes the guiding-center Jacobian $\mathcal{J}_{\text {gc }}$, and summation over particle species is implied whenever an integral over $F_{\mathrm{gc}}$ appears. In addition, the guiding-center magnetic field $\mathbf{H}_{\mathrm{gc}} \equiv \mathbf{B}-4 \pi \mathbf{M}_{\mathrm{gc}}$ is defined in terms of the guiding-center magnetization

$$
\mathbf{M}_{\mathrm{gc}} \equiv \int_{P} F_{\mathrm{gc}}\left(-\mu \widehat{\mathrm{b}}+\frac{q}{c} \mathbb{P}_{\|} \cdot \frac{d \mathbf{X}}{d t}\right)
$$

which is expressed in terms of the intrinsic guidingcenter magnetization $-\mu \widehat{\mathrm{b}}$ and the moving guidingcenter electric-dipole moment [15]

$$
\left(\frac{q \widehat{\mathrm{b}}}{\Omega} \times \frac{d \mathbf{X}}{d t}\right) \times \frac{p_{\|} \widehat{\mathrm{b}}}{m c} \equiv \frac{q}{c} \mathbb{P}_{\|} \cdot \frac{d \mathbf{X}}{d t}
$$

where we introduced the symmetric dyadic tensor

$$
\mathbb{P}_{\|} \equiv \frac{c p_{\|}}{q B}(\mathbf{I}-\widehat{\mathrm{b}} \hat{\mathrm{b}})
$$


We note that, while the guiding-center magnetization (22) is derived from the guiding-center Lagrangian (11): $\mathbf{M}_{\mathrm{gc}} \equiv \int_{P} F_{\mathrm{gc}} \delta L_{\mathrm{gc}} / \delta \mathbf{B}$, the guiding-center polarization $\mathbf{P}_{\mathrm{gc}} \equiv \int_{P} F_{\mathrm{gc}} \delta L_{\mathrm{gc}} / \delta \mathbf{E} \equiv 0$ is absent in our model [16].

We now express Eqs. (19)-21) in Hamiltonian form $\partial \Psi_{\mathrm{gc}}^{a} / \partial t \equiv \mathrm{J}_{\mathrm{gc}}^{a b} \circ \delta \mathcal{H}_{\mathrm{gc}} / \delta \Psi_{\mathrm{gc}}^{b}$ :

$$
\begin{aligned}
\frac{\partial F_{\mathrm{gc}}}{\partial t}= & -B_{\|}^{*}\left\{\frac{F_{\mathrm{gc}}}{B_{\|}^{*}}, \frac{\delta \mathcal{H}_{\mathrm{gc}}}{\delta F_{\mathrm{gc}}}\right\}_{\mathrm{gc}} \\
& -4 \pi q \frac{\delta^{\star} \mathcal{H}_{\mathrm{gc}}}{\delta \mathbf{E}} \cdot B_{\|}^{*}\left\{\mathbf{X}, \frac{F_{\mathrm{gc}}}{B_{\|}^{*}}\right\}_{\mathrm{gc}}, \\
\frac{\partial \mathbf{E}}{\partial t}= & 4 \pi c \nabla \times\left(\frac{\delta \mathcal{H}_{\mathrm{gc}}}{\delta \mathbf{B}}-\frac{q}{c} \int_{P} F_{\mathrm{gc}} \mathbb{P}_{\|} \cdot \frac{d \mathbf{X}}{d t}\right) \\
& -4 \pi q \int_{P} F_{\mathrm{gc}} \frac{d \mathbf{X}}{d t}, \\
\frac{\partial \mathbf{B}}{\partial t}= & -4 \pi c \nabla \times \frac{\delta \mathcal{H}_{\mathrm{gc}}}{\delta \mathbf{E}},
\end{aligned}
$$

where the guiding-center velocity in Eq. (26) is

$$
\frac{d \mathbf{X}}{d t}=\left\{\mathbf{X}, \frac{\delta \mathcal{H}_{\mathrm{gc}}}{\delta F_{\mathrm{gc}}}\right\}_{\mathrm{gc}}+4 \pi q \frac{\delta^{\star} \mathcal{H}_{\mathrm{gc}}}{\delta \mathbf{E}} \cdot\{\mathbf{X}, \mathbf{X}\}_{\mathrm{gc}}
$$

Here, the guiding-center Hamiltonian functional is

$$
\mathcal{H}_{\mathrm{gc}} \equiv \int_{\mathbf{Z}} F_{\mathrm{gc}} K_{\mathrm{gc}}+\int_{\mathbf{X}} \frac{1}{8 \pi}\left(|\mathbf{E}|^{2}+|\mathbf{B}|^{2}\right),
$$

where $\int_{\mathbf{Z}}$ denotes an integration over the guiding-center phase space while $\int_{\mathbf{X}}$ denotes an integral over the threedimensional guiding-center configuration space, from which we obtain the functional derivatives

$$
\left(\begin{array}{c}
\delta \mathcal{H}_{\mathrm{gc}} / \delta F_{\mathrm{gc}} \\
\delta \mathcal{H}_{\mathrm{gc}} / \delta \mathbf{E} \\
\delta \mathcal{H}_{\mathrm{gc}} / \delta \mathbf{B}
\end{array}\right)=\left(\begin{array}{c}
K_{\mathrm{gc}} \\
\mathbf{E} / 4 \pi \\
\mathbf{B} / 4 \pi+\int_{P} F_{\mathrm{gc}} \mu \widehat{\mathrm{b}}
\end{array}\right)
$$

where $\delta K_{\mathrm{gc}} / \delta \mathbf{B}=\mu \widehat{\mathrm{b}}$ and we introduced the definition

$$
\begin{aligned}
4 \pi q \frac{\delta^{\star} \mathcal{H}_{\mathrm{gc}}}{\delta \mathbf{E}} & \equiv 4 \pi q\left(\frac{\delta \mathcal{H}_{\mathrm{gc}}}{\delta \mathbf{E}}+\mathbb{P}_{\|} \cdot \nabla \times \frac{\delta \mathcal{H}_{\mathrm{gc}}}{\delta \mathbf{E}}\right) \\
& =q \mathbf{E}-p_{\|} \frac{\partial \widehat{\mathrm{b}}}{\partial t} \equiv q \mathbf{E}^{*}
\end{aligned}
$$

after making use of Faraday's law (27). We note that the additional guiding-center Maxwell equations are

$$
\begin{aligned}
& \nabla \cdot \mathbf{E}=4 \pi q \int_{P} F_{\mathrm{gc}} \equiv 4 \pi \varrho_{\mathrm{gc}}, \\
& \nabla \cdot \mathbf{B}=0,
\end{aligned}
$$

where Eq. (32) is connected to Eq. (20) through the guiding-center charge conservation law $\partial \varrho_{\mathrm{gc}} / \partial t+$ $\nabla \cdot \mathbf{J}_{\mathrm{gc}}=0$, while Eq. (33) can be viewed as an initial condition of the Faraday equation (21).

\section{GUIDING-CENTER VLASOV-MAXWELL BRACKET}

The guiding-center Vlasov-Maxwell bracket is now constructed from the guiding-center Vlasov-Maxwell equations (25)-(27) and the Hamiltonian functional (29):

$$
\begin{aligned}
\frac{\partial \mathcal{F}}{\partial t} & =\int_{\mathbf{Z}} \frac{\partial F_{\mathrm{gc}}}{\partial t} \frac{\delta \mathcal{F}}{\delta F_{\mathrm{gc}}}+\int_{\mathbf{X}}\left(\frac{\partial \mathbf{E}}{\partial t} \cdot \frac{\delta \mathcal{F}}{\delta \mathbf{E}}+\frac{\partial \mathbf{B}}{\partial t} \cdot \frac{\delta \mathcal{F}}{\delta \mathbf{B}}\right) \\
& \equiv\left\langle\frac{\delta \mathcal{F}}{\delta \Psi^{a}} \mid \frac{\partial \Psi^{a}}{\partial t}\right\rangle=\left\langle\frac{\delta \mathcal{F}}{\delta \Psi^{a}} \mid \mathrm{J}_{\mathrm{gc}}^{a b} \circ \frac{\delta \mathcal{H}_{\mathrm{gc}}}{\delta \Psi^{b}}\right\rangle,
\end{aligned}
$$

where the guiding-center Vlasov-Maxwell bracket for two arbitrary functionals $(\mathcal{F}, \mathcal{G})$ of the fields $\boldsymbol{\Psi}=\left(F_{\mathrm{gc}}, \mathbf{E}, \mathbf{B}\right)$ is defined in terms of the Poisson structure:

$$
[\mathcal{F}, \mathcal{G}]_{\mathrm{gc}} \equiv\left\langle\frac{\delta \mathcal{F}}{\delta \Psi^{a}} \mid \mathrm{J}_{\mathrm{gc}}^{a b} \circ \frac{\delta \mathcal{G}}{\delta \Psi^{b}}\right\rangle .
$$

Here, the antisymmetric Poisson operator $\mathrm{J}_{\mathrm{gc}}^{a b}(\boldsymbol{\Psi})$ guarantees the antisymmetry property: $[\mathcal{F}, \mathcal{G}]_{\mathrm{gc}}=-[\mathcal{G}, \mathcal{F}]_{\mathrm{gc}}$; and the bilinearity of Eq. (35) guarantees the Leibniz property: $[\mathcal{F}, \mathcal{G} \mathcal{K}]_{\mathrm{gc}}=[\mathcal{F}, \mathcal{G}]_{\mathrm{gc}} \mathcal{K}+\mathcal{G}[\mathcal{F}, \mathcal{K}]_{\mathrm{gc}}$. The Jacobi property:

$$
\begin{aligned}
\mathcal{J} a c[\mathcal{F}, \mathcal{G}, \mathcal{K}] \equiv & {\left[[\mathcal{F}, \mathcal{G}]_{\mathrm{gc}}, \mathcal{K}\right]_{\mathrm{gc}}+\left[[\mathcal{G}, \mathcal{K}]_{\mathrm{gc}}, \mathcal{F}\right]_{\mathrm{gc}} } \\
& +\left[[\mathcal{K}, \mathcal{F}]_{\mathrm{gc}}, \mathcal{G}\right]_{\mathrm{gc}}=0
\end{aligned}
$$

which holds for arbitrary functionals $(\mathcal{F}, \mathcal{G}, \mathcal{K})$, involves the guiding-center Poisson operator $\mathrm{J}_{\mathrm{gc}}^{a b}(\boldsymbol{\Psi})$.

From Eq. (34), we can now extract the guiding-center Vlasov-Maxwell bracket expressed in terms of two arbitrary guiding-center functionals $(\mathcal{F}, \mathcal{G})$ as

$$
\begin{aligned}
{[\mathcal{F}, \mathcal{G}]_{\mathrm{gc}}=} & \int_{\mathbf{Z}} F_{\mathrm{gc}}\left\{\frac{\delta \mathcal{F}}{\delta F_{\mathrm{gc}}}, \frac{\delta \mathcal{G}}{\delta F_{\mathrm{gc}}}\right\}_{\mathrm{gc}} \\
& +4 \pi q \int_{\mathbf{Z}} F_{\mathrm{gc}} \frac{\delta^{\star} \mathcal{G}}{\delta \mathbf{E}} \cdot\left\{\mathbf{X}, \frac{\delta \mathcal{F}}{\delta F_{\mathrm{gc}}}\right\}_{\mathrm{gc}} \\
& -4 \pi q \int_{\mathbf{Z}} F_{\mathrm{gc}} \frac{\delta^{\star} \mathcal{F}}{\delta \mathbf{E}} \cdot\left\{\mathbf{X}, \frac{\delta \mathcal{G}}{\delta F_{\mathrm{gc}}}\right\}_{\mathrm{gc}} \\
& +(4 \pi q)^{2} \int_{\mathbf{Z}} F_{\mathrm{gc}}\left(\frac{\delta^{\star} \mathcal{F}}{\delta \mathbf{E}} \cdot\{\mathbf{X}, \mathbf{X}\}_{\mathrm{gc}} \cdot \frac{\delta^{\star} \mathcal{G}}{\delta \mathbf{E}}\right) \\
& +4 \pi c \int_{\mathbf{X}}\left(\frac{\delta \mathcal{F}}{\delta \mathbf{E}} \cdot \nabla \times \frac{\delta \mathcal{G}}{\delta \mathbf{B}}-\frac{\delta \mathcal{G}}{\delta \mathbf{E}} \cdot \nabla \times \frac{\delta \mathcal{F}}{\delta \mathbf{B}}\right),
\end{aligned}
$$

where $\delta^{\star}(\cdots) / \delta \mathbf{E}$ is defined in Eq. (31). The guidingcenter bracket (37) is analogous to the Vlasov-Maxwell bracket (9), where the Maxwell sub-bracket (last term) is identical in both cases, while the guiding-center Vlasov sub-bracket (first term) is connected by guiding-center phase-space transformation of the Vlasov sub-bracket in Eq. (9). The Interaction sub-bracket in Eq. (9), proportional to $4 \pi q$, is transformed into the guiding-center Interaction sub-brackets in Eq. (37), where the quadratic term involving the antisymmetric dyadic Poisson bracket 
$\{\mathbf{X}, \mathbf{X}\}_{\text {gc }}$ represents the moving electric-dipole contribution to guiding-center magnetization, which is absent in Eq. (9). We note that, in contrast to recent work of Burby 17, 18], the guiding-center Vlasov-Maxwell bracket (37) is expressed in terms of functional derivatives involving the Vlasov-Maxwell fields $\left(F_{\mathrm{gc}}, \mathbf{E}, \mathbf{B}\right)$ since there is no guiding-center polarization in our model.

\section{A. Guiding-center momentum conservation law}

The conservation laws of energy-momentum and angular momentum for the guiding-center Vlasov-Maxwell (25) - 27) were recently derived by Brizard and Tronci [12]. As an application of the guiding-center VlasovMaxwell bracket (37), we explore the time derivative of the guiding-center Vlasov-Maxwell (vector-valued) momentum functional

$$
\mathcal{P}_{\mathrm{gc}} \equiv \int_{\mathbf{X}} \mathbf{P}_{\mathrm{gc}}=\int_{\mathbf{Z}} F_{\mathrm{gc}} p_{\|} \widehat{\mathbf{b}}+\int_{\mathbf{X}} \frac{\mathbf{E} \times \mathbf{B}}{4 \pi c}
$$

where each component $\mathcal{P}_{\mathrm{gc}}^{z} \equiv \int_{\mathbf{X}} \mathbf{P}_{\mathrm{gc}} \cdot \widehat{\mathbf{z}}$ satisfies the functional evolution equation

$$
\begin{aligned}
\frac{\partial \mathcal{P}_{\mathrm{gc}}^{z}}{\partial t}= & {\left[\mathcal{P}_{\mathrm{gc}}^{z}, \mathcal{H}_{\mathrm{gc}}\right]_{\mathrm{gc}} } \\
= & \int_{\mathbf{Z}} F_{\mathrm{gc}}\left\{\frac{\delta \mathcal{P}_{\mathrm{gc}}^{z}}{\delta F_{\mathrm{gc}}}, \frac{\delta \mathcal{H}_{\mathrm{gc}}}{\delta F_{\mathrm{gc}}}\right\}_{\mathrm{gc}} \\
& -4 \pi q \int_{\mathbf{Z}} F_{\mathrm{gc}} \frac{\delta^{\star} \mathcal{P}_{\mathrm{gc}}^{z}}{\delta \mathbf{E}} \cdot\left\{\mathbf{X}, \frac{\delta \mathcal{H}_{\mathrm{gc}}}{\delta F_{\mathrm{gc}}}\right\}_{\mathrm{gc}} \\
& +4 \pi q \int_{\mathbf{Z}} F_{\mathrm{gc}} \frac{\delta^{\star} \mathcal{H}_{\mathrm{gc}}}{\delta \mathbf{E}} \cdot\left\{\mathbf{X}, \frac{\delta \mathcal{P}_{\mathrm{gc}}^{z}}{\delta F_{\mathrm{gc}}}\right\}_{\mathrm{gc}} \\
& +(4 \pi q)^{2} \int_{\mathbf{Z}} F_{\mathrm{gc}} \frac{\delta^{\star} \mathcal{P}_{\mathrm{gc}}^{z}}{\delta \mathbf{E}} \cdot\{\mathbf{X}, \mathbf{X}\}_{\mathrm{gc}} \cdot \frac{\delta^{\star} \mathcal{H}_{\mathrm{gc}}}{\delta \mathbf{E}} \\
& +4 \pi c \int_{\mathbf{X}} \frac{\delta \mathcal{H}_{\mathrm{gc}}}{\delta \mathbf{B}} \cdot \nabla \times \frac{\delta \mathcal{P}_{\mathrm{gc}}^{z}}{\delta \mathbf{E}} \\
& -4 \pi c \int_{\mathbf{X}} \frac{\delta \mathcal{P}_{\mathrm{gc}}^{z}}{\delta \mathbf{B}} \cdot \nabla \times \frac{\delta \mathcal{H}_{\mathrm{gc}}}{\delta \mathbf{E}} .
\end{aligned}
$$

Here, the functional derivatives of the guiding-center Hamiltonian functional (29) are given in Eq. (30), and the functional derivatives of the $z$-component of the guidingcenter Vlasov-Maxwell momentum (38) are

$$
\left(\begin{array}{c}
\delta \mathcal{P}_{\mathrm{gc}}^{z} / \delta F_{\mathrm{gc}} \\
4 \pi c \delta \mathcal{P}_{\mathrm{gc}}^{z} / \delta \mathbf{E} \\
4 \pi c \delta \mathcal{P}_{\mathrm{gc}}^{z} / \delta \mathbf{B}
\end{array}\right)=\left(\begin{array}{c}
p_{\|} b_{z} \\
\mathbf{B} \times \widehat{\mathbf{z}} \\
\widehat{\mathbf{z}} \times \mathbf{E}+4 \pi q \int_{P} F_{\mathrm{gc}} \widehat{\mathbf{z}} \cdot \mathbb{P}_{\|}
\end{array}\right)
$$

and

$$
\begin{aligned}
4 \pi c \frac{\delta^{\star} \mathcal{P}_{\mathrm{gc}}^{z}}{\delta \mathbf{E}} & =\mathbf{B} \times \widehat{\mathbf{z}}+\mathbb{P}_{\|} \cdot \nabla \times(\mathbf{B} \times \widehat{\mathbf{z}}) \\
& =\mathbf{B}^{*} \times \widehat{\mathbf{z}}+\left(p_{\|} c / q\right) \nabla b_{z} .
\end{aligned}
$$

In Eq. (39), we now evaluate

$$
\begin{aligned}
& 4 \pi q \frac{\delta^{\star} \mathcal{P}_{\mathrm{gc}}^{z}}{\delta \mathbf{E}} \cdot\left\{\mathbf{X}, K_{\mathrm{gc}}\right\}_{\mathrm{gc}}=\widehat{\mathbf{z}} \cdot \nabla K_{\mathrm{gc}}+\left\{\frac{\delta \mathcal{P}_{\mathrm{gc}}^{z}}{\delta F_{\mathrm{gc}}}, K_{\mathrm{gc}}\right\}_{\mathrm{gc}} \\
& 4 \pi q \frac{\delta^{\star} \mathcal{P}_{\mathrm{gc}}^{z}}{\delta \mathbf{E}} \cdot\{\mathbf{X}, \mathbf{X}\}_{\mathrm{gc}}=\widehat{\mathbf{z}}-\left\{\mathbf{X}, \frac{\delta \mathcal{P}_{\mathrm{gc}}^{z}}{\delta F_{\mathrm{gc}}}\right\}_{\mathrm{gc}},
\end{aligned}
$$

so that

$$
\begin{aligned}
& 4 \pi q \frac{\delta^{\star} \mathcal{H}_{\mathrm{gc}}}{\delta \mathbf{E}} \cdot\left(\left\{\mathbf{X}, \frac{\delta \mathcal{P}_{\mathrm{gc}}^{z}}{\delta F_{\mathrm{gc}}}\right\}_{\mathrm{gc}}+4 \pi q \frac{\delta^{\star} \mathcal{P}_{\mathrm{gc}}^{z}}{\delta \mathbf{E}} \cdot\{\mathbf{X}, \mathbf{X}\}_{\mathrm{gc}}\right) \\
& =q \widehat{\mathbf{z}} \cdot\left(\mathbf{E}+\mathbb{P}_{\|} \cdot \nabla \times \mathbf{E}\right) \equiv q \widehat{\mathbf{z}} \cdot \mathbf{E}^{*}
\end{aligned}
$$

and, hence, we find

$$
\begin{aligned}
\frac{\partial \mathcal{P}_{\mathrm{gc}}^{z}}{\partial t}= & \int_{\mathbf{Z}} F_{\mathrm{gc}}\left[\widehat{\mathbf{z}} \cdot\left(q \mathbf{E}^{*}-\nabla K_{\mathrm{gc}}\right)\right] \\
& +4 \pi c \int_{\mathbf{X}} \frac{\delta \mathcal{H}_{\mathrm{gc}}}{\delta \mathbf{B}} \cdot \nabla \times \frac{\delta \mathcal{P}_{\mathrm{gc}}^{z}}{\delta \mathbf{E}} \\
& -4 \pi c \int_{\mathbf{X}} \frac{\delta \mathcal{P}_{\mathrm{gc}}^{z}}{\delta \mathbf{B}} \cdot \nabla \times \frac{\delta \mathcal{H}_{\mathrm{gc}}}{\delta \mathbf{E}} .
\end{aligned}
$$

Next, we find

$$
\begin{aligned}
4 \pi c \frac{\delta \mathcal{H}_{\mathrm{gc}}}{\delta \mathbf{B}} \cdot \nabla \times \frac{\delta \mathcal{P}_{\mathrm{gc}}^{z}}{\delta \mathbf{E}}= & \nabla \cdot\left(\frac{|\mathbf{B}|^{2}}{8 \pi} \widehat{\mathrm{z}}\right)+\int_{P} F_{\mathrm{gc}} \widehat{\mathbf{z}} \cdot \nabla K_{\mathrm{gc}} \\
4 \pi c \frac{\delta \mathcal{P}_{\mathrm{gc}}^{z}}{\delta \mathbf{B}} \cdot \nabla \times \frac{\delta \mathcal{H}_{\mathrm{gc}}}{\delta \mathbf{E}}= & -\nabla \cdot\left[\left(\frac{\mathbf{E E}}{4 \pi}-\frac{|\mathbf{E}|^{2}}{8 \pi} \mathbf{I}\right) \cdot \widehat{\mathrm{z}}\right] \\
& +\int_{P} F_{\mathrm{gc}} q \mathbf{E}^{*} \cdot \widehat{\mathrm{z}}
\end{aligned}
$$

where we made use of Eq. (32), so that Eq. (39) yields the guiding-center Vlasov-Maxwell momentum conservation law $\partial \mathcal{P}_{\text {gc }} / \partial t=\left[\mathcal{P}_{\text {gc }}, \mathcal{H}_{\text {gc }}\right]_{\text {gc }}=0$. The derivation of the angular guiding-center momentum conservation law $\partial \mathcal{P}_{\text {gc } \varphi} / \partial t=\left[\mathcal{P}_{\text {gc } \varphi}, \mathcal{H}_{\mathrm{gc}}\right]_{\mathrm{gc}}=0$, where $\mathcal{P}_{\mathrm{gc} \varphi}=$ $\int_{\mathbf{X}} \mathbf{P}_{\mathrm{gc}} \cdot \partial \mathbf{X} / \partial \varphi$ follows similar steps.

\section{B. Guiding-center Casimir functionals}

Casimir functionals $\mathcal{C}$ satisfy the bracket property $[\mathcal{C}, \mathcal{K}]_{\text {gc }}=0$, which holds for an arbitrary functional $\mathcal{K}$. A standard example is the guiding-center entropy functional (omitting Boltzmann's constant)

$$
\mathcal{S}_{\mathrm{gc}}\left[F_{\mathrm{gc}}, \mathbf{B}\right] \equiv-\int_{\mathbf{Z}} F_{\mathrm{gc}} \ln \left(F_{\mathrm{gc}} / B_{\|}^{*}\right),
$$

for which we obtain

$$
\begin{aligned}
{\left[\mathcal{S}_{\mathrm{gc}}, \mathcal{K}\right]_{\mathrm{gc}}=} & \int_{\mathrm{Z}} F_{\mathrm{gc}}\left\{\frac{\delta \mathcal{S}_{\mathrm{gc}}}{\delta F_{\mathrm{gc}}}, \frac{\delta \mathcal{K}}{\delta F_{\mathrm{gc}}}\right\}_{\mathrm{gc}} \\
& +4 \pi q \int_{\mathbf{Z}} F_{\mathrm{gc}} \frac{\delta^{\star} \mathcal{K}}{\delta \mathbf{E}} \cdot\left\{\mathbf{X}, \frac{\delta \mathcal{S}_{\mathrm{gc}}}{\delta F_{\mathrm{gc}}}\right\}_{\mathrm{gc}} \\
& -4 \pi c \int_{\mathbf{X}} \frac{\delta \mathcal{K}}{\delta \mathbf{E}} \cdot \nabla \times \frac{\delta \mathcal{S}_{\mathrm{gc}}}{\delta \mathbf{B}}
\end{aligned}
$$


where $\delta \mathcal{S}_{\mathrm{gc}} / \delta F_{\mathrm{gc}}=-1-\ln (F)$, with $F \equiv F_{\mathrm{gc}} / B_{\|}^{*}$, and

$$
\frac{\delta \mathcal{S}_{\mathrm{gc}}}{\delta \mathbf{B}}=\int_{P}\left[F \widehat{\mathrm{b}}-\frac{q}{c} \mathbb{P}_{\|} \cdot\left(\mathbf{B}^{*} \frac{\partial F}{\partial p_{\|}}+\frac{c \widehat{\mathrm{b}}}{q} \times \nabla F\right)\right],
$$

which is obtained after using the magnetic variations

$$
\left.\begin{array}{rl}
\delta \mathbf{B}^{*} & =\delta \mathbf{B}+\nabla \times\left(\mathbb{P}_{\|} \cdot \delta \mathbf{B}\right) \\
(c / q) \delta \widehat{\mathrm{b}} & =\delta \mathbf{B} \cdot \partial \mathbb{P}_{\|} / \partial p_{\|} \\
\delta B_{\|}^{*} & =\delta \widehat{\mathrm{b}} \cdot \mathbf{B}^{*}+\widehat{\mathrm{b}} \cdot \delta \mathbf{B}^{*}
\end{array}\right\}
$$

Hence, we find the guiding-center bracket identity

$$
\begin{aligned}
{\left[\mathcal{S}_{\mathrm{gc}}, \mathcal{K}\right]_{\mathrm{gc}}=} & -\int_{\mathbf{Z}} B_{\|}^{*}\left\{F, \frac{\delta \mathcal{K}}{\delta F_{\mathrm{gc}}}\right\}_{\mathrm{gc}} \\
& -4 \pi q \int_{\mathbf{Z}} \frac{\delta^{\star} \mathcal{K}}{\delta \mathbf{E}} \cdot\left(\mathbf{B}^{*} \frac{\partial F}{\partial p_{\|}}+\frac{c \widehat{\mathrm{b}}}{q} \times \nabla F\right) \\
& -4 \pi c \int_{\mathbf{X}} \frac{\delta \mathcal{S}_{\mathrm{gc}}}{\delta \mathbf{B}} \cdot \nabla \times \frac{\delta \mathcal{K}}{\delta \mathbf{E}} \equiv 0,
\end{aligned}
$$

where the first term on the right side vanishes since it is an exact phase-space divergence, while the last term cancels out the second term. The expression (43) for the guiding-center Vlasov-Maxwell entropy was recently mentioned by Burby and Tronci [19] and might find applications in the dissipative guiding-center bracket formulation [20 22] of the guiding-center Vlasov-MaxwellLandau model (e.g., see Ref. [23]).

\section{Jacobi property of the guiding-center Vlasov-Maxwell bracket}

We now verify that the guiding-center bracket (37) satisfies the Jacobi property (36). According to the
Bracket theorem [8], the proof of the Jacobi property involves only the explicit dependence of the guiding-center Vlasov-Maxwell bracket (37) on the guiding-center fields $\mathrm{J}_{\mathrm{gc}}^{a b}\left(F_{\mathrm{gc}}, \mathbf{B}\right)$, where we note that the dependence on the magnetic field $\mathbf{B}$ enters through the guiding-center Poisson bracket (14), while the electric field $\mathbf{E}$ is explicitly absent.

Hence, we can write the double-bracket involving three arbitrary guiding-center functionals $(\mathcal{F}, \mathcal{G}, \mathcal{K})$ :

$$
\begin{aligned}
{\left[[\mathcal{F}, \mathcal{G}]_{\mathrm{gc}}, \mathcal{K}\right]_{\mathrm{gc}}^{P}=} & \int_{\mathbf{Z}} F_{\mathrm{gc}}\left\{\frac{\delta^{P}[\mathcal{F}, \mathcal{G}]_{\mathrm{gc}}}{\delta F_{\mathrm{gc}}}, \frac{\delta \mathcal{K}}{\delta F_{\mathrm{gc}}}\right\}_{\mathrm{gc}} \\
& +4 \pi q \int_{\mathbf{Z}} F_{\mathrm{gc}} \frac{\delta^{\star} \mathcal{K}}{\delta \mathbf{E}} \cdot\left\{\mathbf{X}, \frac{\delta^{P}[\mathcal{F}, \mathcal{G}]_{\mathrm{gc}}}{\delta F_{\mathrm{gc}}}\right\}_{\mathrm{gc}} \\
& -4 \pi c \int_{\mathbf{X}} \frac{\delta^{P}[\mathcal{F}, \mathcal{G}]_{\mathrm{gc}}}{\delta \mathbf{B}} \cdot \nabla \times \frac{\delta \mathcal{K}}{\delta \mathbf{E}},
\end{aligned}
$$

where the terms involving $\delta^{P}[\mathcal{F}, \mathcal{G}]_{\mathrm{gc}} / \delta \mathbf{E}$ vanish on the basis of the Bracket theorem. Here, the functional derivative $\delta^{P}[\mathcal{F}, \mathcal{G}]_{\mathrm{gc}} / \delta F_{\mathrm{gc}}$ involves the explicit dependence on the guiding-center Vlasov distribution $F_{\mathrm{gc}}$ in the Vlasov and Interaction sub-brackets, while $\delta^{P}[\mathcal{F}, \mathcal{G}]_{\text {gc }} / \delta \mathbf{B}$ in the Maxwell sub-bracket involves the explicit dependence on the magnetic field $\mathbf{B}$, which appears through $\left(\widehat{\mathrm{b}}, \mathbf{B}^{*}, B_{\|}^{*}\right)$ in the guiding-center Poisson bracket (14) and the dyadic tensor (24), where $\left(\delta \widehat{\mathrm{b}}, \delta \mathbf{B}^{*}, \delta B_{\|}^{*}\right)$ are given in Eq. (45).

The proof of the Jacobi property for the guiding-center bracket (37) involves using several identities derived from the guiding-center Poisson bracket (14) leading to an expansion in powers of $\epsilon \equiv 4 \pi q$ up to third order:

$$
\begin{aligned}
\mathcal{J} a c[\mathcal{F}, \mathcal{G}, \mathcal{K}]= & \int_{\mathbf{Z}} F_{\mathrm{gc}}\left(\left\{\{f, g\}_{\mathrm{gc}}, k\right\}_{\mathrm{gc}}+\left\{\{g, k\}_{\mathrm{gc}}, f\right\}_{\mathrm{gc}}+\left\{\{k, f\}_{\mathrm{gc}}, g\right\}_{\mathrm{gc}}\right) \\
& +\epsilon \int_{\mathbf{Z}} F_{\mathrm{gc}}\left[F_{i}^{\star}\left(\left\{\left\{X^{i}, g\right\}_{\mathrm{gc}}, k\right\}_{\mathrm{gc}}+\left\{\{g, k\}_{\mathrm{gc}}, X^{i}\right\}_{\mathrm{gc}}+\left\{\left\{k, X^{i}\right\}_{\mathrm{gc}}, g\right\}_{\mathrm{gc}}\right)+\circlearrowleft\right] \\
& +\epsilon^{2} \int_{\mathbf{Z}} F_{\mathrm{gc}}\left[F_{i}^{\star} G_{j}^{\star}\left(\left\{\left\{X^{i}, X^{j}\right\}_{\mathrm{gc}}, k\right\}_{\mathrm{gc}}+\left\{\left\{X^{j}, k\right\}_{\mathrm{gc}}, X^{i}\right\}_{\mathrm{gc}}+\left\{\left\{k, X^{i}\right\}_{\mathrm{gc}}, X^{j}\right\}_{\mathrm{gc}}\right)+\circlearrowleft\right] \\
& +\epsilon^{3} \int_{\mathbf{z}} F_{\mathrm{gc}}\left[F_{i}^{\star} G_{j}^{\star} K_{\ell}^{\star}\left(\left\{\left\{X^{i}, X^{j}\right\}_{\mathrm{gc}}, X^{\ell}\right\}_{\mathrm{gc}}+\left\{\left\{X^{j}, X^{\ell}\right\}_{\mathrm{gc}}, X^{i}\right\}_{\mathrm{gc}}+\left\{\left\{X^{\ell}, X^{i}\right\}_{\mathrm{gc}}, X^{j}\right\}_{\mathrm{gc}}\right)\right],
\end{aligned}
$$

where $(f, g, k) \equiv\left(\delta \mathcal{F} / \delta F_{\mathrm{gc}}, \delta \mathcal{G} / \delta F_{\mathrm{gc}}, \delta \mathcal{K} / \delta F_{\mathrm{gc}}\right)$ and $\left(\mathbf{F}^{\star}, \mathbf{G}^{\star}, \mathbf{K}^{\star}\right) \equiv\left(\delta^{\star} \mathcal{F} / \delta \mathbf{E}, \delta^{\star} \mathcal{G} / \delta \mathbf{E}, \delta^{\star} \mathcal{K} / \delta \mathbf{E}\right)$, while all additional terms have cancelled out exactly (details of the proof are presented elsewhere [24]). Here, summation over the repeated indices $(i, j, \ell)$ denoting the compo- nents of the vector fields $\left(\mathbf{F}^{\star}, \mathbf{G}^{\star}, \mathbf{K}^{\star}\right)$ is implied, and the symbol $\circlearrowleft$ denotes cyclic permutations of the functionals $(\mathcal{F}, \mathcal{G}, \mathcal{K})$.

In Eq. (47), it is clear that the Jacobi property of the guiding-center Vlasov-Maxwell bracket (37) is inherited 
from the Jacobi property (16) of the guiding-center Poisson bracket (14), since each term in Eq. (47) vanishes identically because of this latter property. Hence, the Jacobi property for the guiding-center Vlasov-Maxwell bracket (37) holds under the condition (17).

\section{DISCUSSION}

In the present paper, we derived the Hamiltonian structure of the guiding-center Vlasov-Maxwell equations introduced by Brizard and Tronci [12]. The associated guiding-center momentum and angular-momentum conservation laws were also presented in Hamiltonian form in terms of the guiding-center Vlasov-Maxwell bracket (37). Since the guiding-center kinetic energy and Poisson bracket associated with the guiding-center VlasovMaxwell model considered here are both independent of the electric field, the effects of guiding-center polarization only appear through the moving electric-dipole contribution to the guiding-center magnetization (22).

Future work will consider the inclusion of guidingcenter polarization into our guiding-center Vlasov-
Maxwell equations, as well as applications of the guidingcenter Hamiltonian structure in gauge-free gyrokinetic Vlasov-Maxwell theory [25, 26], where the perturbed electromagnetic fields $\left(\mathbf{E}_{1}, \mathbf{B}_{1}\right)$ appear explicitly in the gyrocenter Lagrangian (e.g., see Ref. 27]). We also plan to use the guiding-center Vlasov-Maxwell bracket (37) to explore extensions of Hamiltonian functional perturbation theory [28].

\section{Acknowledgments}

The Author wishes to acknowledge useful discussions with C. Tronci and J.W. Burby. This work was supported by the National Science Foundation grant No. PHY1805164 .

\section{Data Availability Statement}

Data sharing is not applicable to this article as no new data were created or analyzed in this study.
[1] P.J. Morrison, Phys. Plasmas 12, 058102 (2005).

[2] P.J. Morrison, Phys. Plasmas 24, 055502 (2017).

[3] H. Ye and P.J. Morrison, Phys. Fluids B 4, 771 (1992).

[4] A.J. Brizard, Phys. Rev. Lett. 84, 5768 (2000).

[5] P. J. Morrison, Phys. Lett. A80, 383 (1980).

[6] J. E. Marsden, A. Weinstein, Physica 4D, 394 (1982).

[7] I. Bialynicki-Birula, J. C. Hubbard, L. A. Turski, Physica 128A, 509 (1984).

[8] P.J. Morrison, Phys. Plasmas 20, 012104 (2013).

[9] A.J. Brizard, P.J. Morrison, J.W. Burby, L. de Guillebon, and M. Vittot, Lifting of the Vlasov-Maxwell Bracket by Lie-transform Method, arXiv:1606.06652 (2016).

[10] A.J. Brizard, P.J. Morrison, J.W. Burby, L. de Guillebon, and M. Vittot, J. Plasma Phys. 82, 905820608 (2016).

[11] D. Pfirsch and P.J. Morrison, Phys. Rev. A 32, 1714 (1985).

[12] A.J. Brizard and C. Tronci, Phys. Plasmas 23, 062107 (2016).

[13] R.G. Littlejohn, J. Plasma Phys. 29, 111 (1983).

[14] J.R. Cary and A.J. Brizard, Rev. Mod. Phys. 81, 693 (2009).

[15] N. Tronko and A.J. Brizard, Phys. Plasmas 22, 112507 (2015).

[16] In Ref. 11], the guiding-center Lagrangian (11) is replaced with $L_{\mathrm{gc}}=\left(q \mathbf{A} / c+\boldsymbol{\Pi}_{\mathrm{gc}}\right) \cdot d \mathbf{X} / d t-(q \Phi+$ $\left.K_{\mathrm{gc}}\right)$, where $\boldsymbol{\Pi}_{\mathrm{gc}} \equiv p_{\|} \widehat{\mathrm{b}}+\mathbf{E} \times q \widehat{\mathrm{b}} / \Omega$ and $K_{\mathrm{gc}}=$ $\mu B+\left|\boldsymbol{\Pi}_{\mathrm{gc}}\right|^{2} / 2 m$. Hence, the guiding-center electric- dipole moment is $\delta L_{\mathrm{gc}} / \delta \mathbf{E}=\left(\delta \mathbf{\Pi}_{\mathrm{gc}} / \delta \mathbf{E}\right) \cdot(d \mathbf{X} / d t-$ $\left.\boldsymbol{\Pi}_{\mathrm{gc}} / m\right)=(q \widehat{\mathbf{b}} / \Omega) \times(d \mathbf{X} / d t-\mathbf{E} \times c \widehat{\mathrm{b}} / B)$ and the guiding-center magnetic-dipole moment is $\delta L_{\mathrm{gc}} / \delta \mathbf{B}=$ $-\mu \widehat{\mathrm{b}}+\left(\delta \boldsymbol{\Pi}_{\mathrm{gc}} / \delta \mathbf{B}\right) \cdot\left(d \mathbf{X} / d t-\boldsymbol{\Pi}_{\mathrm{gc}} / m\right)$. In addition, the effective fields (15) are replaced with $q \mathbf{E}^{* *} \equiv q \mathbf{E}-$ $\partial \boldsymbol{\Pi}_{\mathrm{gc}} / \partial t, \mathbf{B}^{* *} \equiv \mathbf{B}+\nabla \times\left(c \boldsymbol{\Pi}_{\mathrm{gc}} / q\right)$, and $B_{\|}^{* *} \equiv \widehat{\mathrm{b}} \cdot \mathbf{B}^{* *}$.

[17] J.W. Burby, Chasing Hamiltonian structure in gyrokinetic theory, arXiv:1509.04551 (2015).

[18] J.W. Burby, Phys. Plasmas 24, 032101 (2017).

[19] J.W. Burby and C. Tronci, Plasma Phys. Control. Fusion 59, 045013 (2017).

[20] A.N. Kaufman, Phys. Lett. 100A, 419 (1984).

[21] P.J. Morrison, Phys. Lett. 100A, 423 (1984).

[22] P.J. Morrison, Physica 18D, 410 (1986).

[23] R. Iorio and E. Hirvijoki, J. Plasma Phys. 87, 835870401 (2021).

[24] A. J. Brizard, Proof of the Jacobi property of the guidingcenter Vlasov-Maxwell bracket, arXiv:2107.08129 (2021).

[25] J.W. Burby and A.J. Brizard, Phys. Lett. A 383, 2172 (2019).

[26] A.J. Brizard, J. Plasma Phys. 87, 905870307 (2021).

[27] A.J. Brizard, Hamiltonian structure of the gauge-free gyrokinetic Vlasov-Maxwell equations, arXiv:2108.09384 (2021).

[28] A.J. Brizard and C. Chandre, Phys. Plasmas 27, 122111 (2020). 